\title{
Dynamics of BMP and Hes1/Hairy1 signaling in the dorsal neural tube underlies the transition from neural crest to definitive roof plate
}

\author{
Erez Nitzan ${ }^{1,2+}$, Oshri Avraham ${ }^{1,3 \dagger}$, Nitza Kahane ${ }^{1}$, Shai Ofek¹, Deepak Kumar ${ }^{1}$ and Chaya Kalcheim ${ }^{1 *}$
}

\begin{abstract}
Background: The dorsal midline region of the neural tube that results from closure of the neural folds is generally termed the roof plate (RP). However, this domain is highly dynamic and complex, and is first transiently inhabited by prospective neural crest (NC) cells that sequentially emigrate from the neuroepithelium. It only later becomes the definitive RP, the dorsal midline cells of the spinal cord. We previously showed that at the trunk level of the axis, prospective RP progenitors originate ventral to the premigratory NC and progressively reach the dorsal midline following NC emigration. However, the molecular mechanisms underlying the end of NC production and formation of the definitive RP remain virtually unknown.

Results: Based on distinctive cellular and molecular traits, we have defined an initial NC and a subsequent RP stage, allowing us to investigate the mechanisms responsible for the transition between the two phases.

We demonstrate that in spite of the constant production of BMP4 in the dorsal tube at both stages, RP progenitors only transiently respond to the ligand and lose competence shortly before they arrive at their final location. In addition, exposure of dorsal tube cells at the NC stage to high levels of BMP signaling induces premature RP traits, such as Hes 1/ Hairy 1, while concomitantly inhibiting NC production. Reciprocally, early inhibition of BMP signaling prevents Hairy 1 mRNA expression at the RP stage altogether, suggesting that BMP is both necessary and sufficient for the development of this RP-specific trait.

Furthermore, when Hes1/Hairy1 is misexpressed at the NC stage, it inhibits BMP signaling and downregulates BMPR1A Alk3 mRNA expression, transcription of BMP targets such as Foxd3, cell-cycle progression, and NC emigration. Reciprocally, Foxd3 inhibits Hairy1, suggesting that repressive cross-interactions at the level of, and downstream from, BMP ensure the temporal separation between both lineages.
\end{abstract}

Conclusions: Together, our data suggest that BMP signaling is important both for NC and RP formation. Given that these two structures develop sequentially, we speculate that the longer exposure of RP progenitors to BMP compared with that of premigratory NC cells may be translated into a higher signaling level in the former. This induces changes in responsiveness to BMP, most likely by downregulating the expression of Alk3 receptors and, consequently, of BMPdependent downstream transcription factors, which exhibit spatial complementary expression patterns and mutually repress each other to generate alternative fates. This molecular dynamic is likely to account for the transition between the $\mathrm{NC}$ and definitive RP stages and thus be responsible for the segregation between central and peripheral lineages during neural development.

Keywords: Avian embryo, BMP, Epithelial-to-mesenchymal transition, Foxd3, Hairy1, HES1, Dorsal interneurons, Dorso-ventral patterning, Neural tube

\footnotetext{
* Correspondence: kalcheim@cc.huji.ac.il

${ }^{\dagger}$ Equal contributors

'Department of Medical Neurobiology, IMRIC and ELSC, Hebrew University of Jerusalem-Hadassah Medical School, Jerusalem 9112102, PO Box 12272, Israel

Full list of author information is available at the end of the article
} 


\section{Background}

The fundamental decision whether to become peripheral nervous system (PNS) or central nervous system (CNS) takes place in the dorsal domain of the developing neural tube (NT). In this area three main populations of cells are sequentially generated. First to appear are various populations of neural crest (NC) cells, consisting of precursors of most neurons and all glia of the PNS as well as melanocytes, endocrine derivatives, etc. [1-3]. Second to appear are roof plate (RP) cells, which constitute the definitive dorsal midline of the CNS, and third are dorsal spinal interneurons, whose specification and differentiation are controlled by dorsal NT-derived signals [4-8].

Only selected aspects of dorsal NT development have been experimentally addressed, and many essential questions remain unanswered. For instance, the mechanisms responsible for the ordered transition between $\mathrm{NC}$ and $\mathrm{RP}$ are largely unknown. Although prospective premigratory NC and RP cells are not distinguishable at the early stages either morphologically or by molecular means, NC progenitors proliferate both before as well as after delamination, and in the trunk they synchronously exit the epithelium at the $S$ phase of the cell cycle [9]. In contrast, RP cells become post-mitotic [10] and adopt their characteristic morphology, constituting the definitive dorsal domain of the spinal cord.

Single cell lineage tracing suggested that NC and NT progenitors derive from a common founder cell [11]. Nonetheless, these studies did not identify the precise CNS cell types involved (i.e., RP, specific interneurons, or both), neither did they determine the exact stage(s) in which this putative common founder cell prevails or segregates. Hence, questions remain, such as where in the dorsal NT do RP cells originate and when precisely do they segregate from the NC lineage? Using spatiotemporally controlled lineage analysis, our previous findings revealed that the dorsal NT is sequentially transited by distinct cell populations that exit the NT to populate NC derivatives $[12,13]$. In one of these studies it was found that RP progenitors originate ventral to the premigratory $\mathrm{NC}$ and relocate ventro-dorsally to reach their final position in the dorsal midline of the NT upon completion of NC exit [12]. Furthermore, tracing the dynamics of the NC marker Foxd3 using a specific reporter revealed that $\mathrm{NC}$ and $\mathrm{RP}$ progenitors are initially part of the Foxd3 lineage, yet RP precursors downregulate its expression while relocating into the dorsal midline; this occurs when some NC progenitors still reside within the neuroepithelium [13]. Hence, it is most likely that RP precursors share a common lineage with $\mathrm{NC}$ only at an early stage of dorsal NT development. This domain is, therefore, a dynamic area in which progressive $\mathrm{NC}$ emigration takes place until replacement by the definitive
$\mathrm{RP}$, thus resulting in the separation between CNS and PNS [4, 12].

The signaling activity of bone morphogenetic proteins (BMPs) is essential for NC induction, epithelial-tomesenchymal transitions (EMTs), and specification and patterning of dorsal interneurons [6, 8, 14-19]. But whether BMP signaling is necessary for RP development and what distinguishes between $\mathrm{RP}$ and $\mathrm{NC}$ in terms of BMP activity remains to be addressed. The transcription factors Lmx1a and 1b are highly expressed in the dorsal NT and differentiated RP cells in the chick developing spinal cord. They were reported to act downstream to BMP and to affect expression of RP markers and interneuron development $[20,21]$. However, given that Lmx genes in turn stimulate expression of both BMP and Wnt $[20,21]$, the possibility exists that BMP acts on RP and/or interneuron production independently of Lmx or that additional downstream factors are necessary.

In the present study, we characterize the nascent RP as an epithelial group of cells exhibiting apico-basal polarity and apically localized cilia, little or no cell proliferation, expression and activity of the basic helixloop-helix (bHLH) transcriptional repressor Hes1/Hairy1 $[22,23]$, and transcription of the ciliary protein Foxj1 [24]. This contrasts with the proliferative NC stage in which dorsal NT cells are devoid of, or discontinuously express, N-cadherin and laminin while transcribing typical NC markers such as Foxd3, Snail2, and Sox9 [4, 12, 13]. Furthermore, we show that despite the constant production of BMP4 in the dorsal NT, RP progenitors only initially respond to the ligand and lose competence during their ventro-dorsal relocation towards the midline of the NT, with a concomitant downregulation of BMPR1A/Alk3. Because both $\mathrm{NC}$ and RP progenitors are localized within a domain of high BMP activity [18], we hypothesized that RP progenitors are also sensitive to BMP signaling. Indeed, constitutive activation of the BMP pathway resulted in premature transcription of the RP marker Hairy1. Notably, this was associated with an inhibition of NC production. Furthermore, early misexpression of Hes1/Hairy1 at the NC stage inhibited BMP signaling while downregulating expression of the Alk3 receptor, transcription of BMP targets such as Foxd3, cell-cycle progression, and NC emigration. Conversely, Foxd3 inhibited Hairy1 altogether, suggesting that repressive cross-interactions at the level of and downstream of BMP ensure the temporal separation between the two lineages.

\section{Results}

Cellular characterization of the dorsal NT at NC and RP stages

To begin understanding the mechanisms underlying the transition from the $\mathrm{NC}$ to the $\mathrm{RP}$ stage, we first 
characterized differences in cellular behavior between the two phases. Following electroporation of a GFP-encoding DNA into the flank-level NT at embryonic day 2 (E2) (Hamburger and Hamilton stages HH12-14, 17-20 somite stage [ss]), we observed labeled $\mathrm{NC}$ derivatives in the periphery at E4.5 (Fig. 1a). GFP+ cells were found in characteristic positions, such as the sympathetic ganglia, along nerves as Schwann cells, in the dorsal root ganglion (DRG), and in the dermis corresponding to melanocytes (see also [12]). In contrast, electroporation of GFP at E3.5

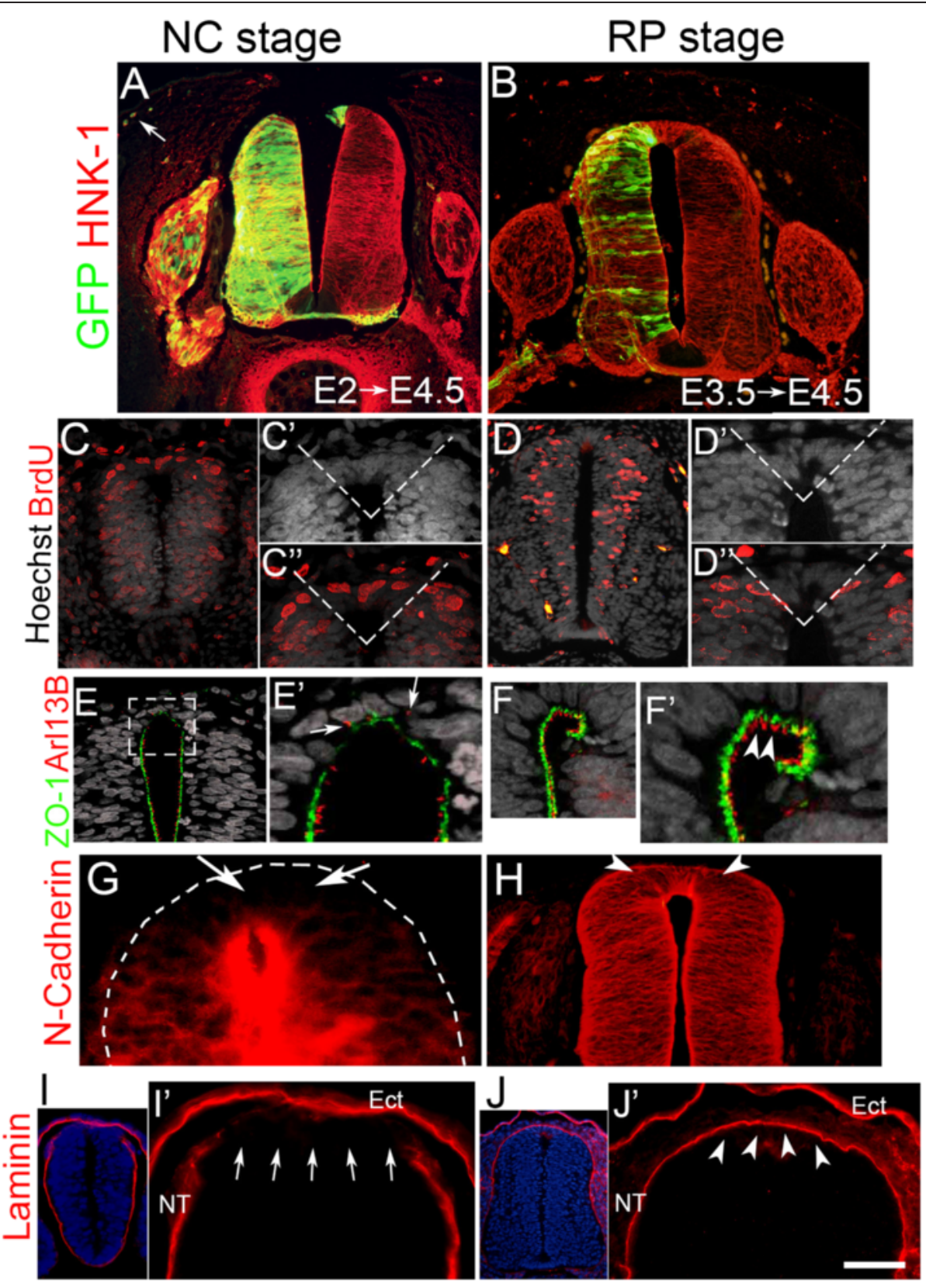

Fig. 1 Differential cellular characteristics of the neural tube at neural crest (NC) and roof plate (RP) stages. $\mathbf{a}, \mathbf{b}$ Transverse sections of the flank region of E4.5 avian embryos whose hemi-neural tubes (NTs) were electroporated with a control GFP plasmid at E2 (a) or E3.5 (b). Note the contribution of labeled cells to NC derivatives including melanocytes (arrow in a) following early but not late stage electroporations. $\mathbf{c}$, $\mathbf{d}$ Bromodeoxyuridine (BrdU) incorporation following a 1-h pulse at NC (E2-E2.5, c) or RP (E3.5, d) stages. Dashed lines in insets mark the dorsal NT domain that was quantified (see text for details). Note the presence of the BrdU+ nuclei (Red) in $\mathbf{c}-\mathbf{c}^{\mathbf{\prime}}$ but not in the equivalent dashed area in $\mathbf{d}-\mathbf{d} \mathbf{d}^{\prime \prime}$. Nuclei were visualized with Hoechst. e-j Antibody staining for epithelial (ZO-1, N-cadherin, laminin) or ciliary (Arl13b) markers. Arrows point to disorganized cilia (e'), the absence of Ncadherin in the dorsal NT compared to more ventral regions ( $\mathbf{g})$, and an incomplete laminin-containing basal lamina (i, i') at the NC stage. In contrast, note the apically oriented cilia (f), positive N-cadherin immunostaining $(\mathbf{h})$, and continuous laminin expression (j) in the dorsal NT at the RP stage (arrowheads in $\mathbf{f}, \mathbf{h}$, and $\left.\mathbf{j}^{\prime}\right)$. Ect ectoderm. Bar in $\mathbf{a}, \mathbf{b}, \mathbf{d}, \mathbf{h}, \mathbf{j}=80 \mu \mathrm{M} ; \mathbf{c}=50 \mu \mathrm{M} ; \mathbf{c}^{\prime}, \mathbf{d}^{\prime}, \mathbf{e}=30 \mu \mathrm{M} ; \mathbf{f}, \mathbf{g}, \mathbf{i}^{\prime}=40 \mu \mathrm{M} ; \mathbf{e}^{\prime}=15 \mu \mathrm{M} ; \mathbf{j}=240 \mu \mathrm{M} ; \mathbf{i}=140 \mu \mathrm{M}$ 
(40 ss, HH19-20) followed by fixation at E4.5 did not produce any labeled cells outside the neuroepithelium (Fig. 1b), in agreement with our previous results [12]. This suggests that by E3.5 at flank levels of the axis, all NC progenitors have already left the dorsal neuroepithelium, which is now occupied by definitive RP cells. Hence, we refer to the period between E2 and E3.5 in which the dorsal NT produces emigrating cells as the "NC stage" and to the period starting from E3.5 onward as the "RP stage."

We next characterized the dynamics of cell proliferation in the dorsal NT, because the transition from the G1 to the $\mathrm{S}$ phase of the cell cycle was found to be critical for NC delamination [9]. To this end, an area containing 810 nuclei per hemi-NT was considered in each section (dashed lines in Fig. 1c, d). These cells are included within the expression domain of Foxd 3 and MafB at the NC and RP stages, respectively. At the NC stage, $45.9 \pm 4.0 \%$ of the cells in the dorsal NT had incorporated BrdU following a 1-h pulse, consistent with previous findings [17]. At more advanced stages of $\mathrm{NC}$ delamination, the proportion of BrdU+ nuclei decreased to $26.4 \pm 3.4 \%$, and by E3.5 only $4.8 \pm 0.5 \%$ of the nuclei were BrdU+ $(\mathrm{N}=5$ embryos/ stage, Fig. 1c, d). Thus, the shift from the NC stage to the RP stage involves cell-cycle exit [10], which is associated with the end of $\mathrm{NC}$ emigration.

Next, we examined the expression of several proteins that characterize embryonic epithelia. At the NC stage, the apical epithelial markers $\mathrm{ZO}-1$ and $\mathrm{N}$-cadherin were discontinuous or virtually absent in the dorsal NT, respectively (Fig. 1e, g) [25]. In addition, Arl13b-positive cilia [26] were randomly distributed in the dorsal NT rather than pointing apically into the NT lumen as observed at more ventral regions of the neuroepithelium (Fig. 1e, e'). Furthermore, the laminin-expressing basement membrane at the basal side of the dorsal NT was discontinuous (Fig. 1i, i', see also [27, 28]). In contrast, a close association between ZO-1 and Arl13b in the apical side of the NT was apparent at the RP stage (Fig. 1f, f'), $\mathrm{N}$-cadherin was re-expressed (Fig. 1h), and the lamininpositive basal lamina was uninterrupted (Fig. 1j, j'). These observations suggest that the nascent RP regains epithelial characteristics such as apico-basal polarity, in spite of these being disrupted during the period of $\mathrm{NC}$ delamination.

\section{Shared and differential transcriptional gene patterns in the dorsal NT at NC and RP stages}

To elucidate the mechanisms leading to the transition between NC and RP stages, it would be useful to identify and functionally characterize genes whose expression is restricted to either stage. To this end, we examined expression patterns of candidate dorsal NT markers.

The Id family of transcriptional regulators encodes four HLH proteins that lack a basic DNA-binding domain, and function in a dominant negative manner by binding and sequestering bHLH transcription factors into inactive heterodimers [29, 30]. Id2 was reported to regulate $\mathrm{NC}$ specification, and to maintain the balance between cell differentiation and proliferation [31]. Ids are also known effectors of BMP signaling [32-34]. Id2 and Id3 were expressed in the dorsal NT at the NC stage, but not in the definitive RP, and at the RP stage $I d 2 / 3$ only appeared ventral to the RP, likely in dorsal interneuron progenitors (Additional file 1: Figure S1A-D). In contrast, no Id1 or Id4 were apparent in the dorsal NT (not shown). Leukocyte tyrosine kinase (LTK) is expressed in zebrafish $\mathrm{NC}$ cells, and in particular in iridophores, where it was shown to be critical for their development [35]. LTK is also expressed in migrating avian cranial $\mathrm{NC}$ [36]. In the trunk, LTK was transcribed in the dorsal NT at the $\mathrm{NC}$ but not RP stage (Additional file 1: Figure S1E, F). Additionally, transcription of Foxd3, Sox9, and Snail2 was restricted to the neural progenitors of the $\mathrm{NC}$ and absent from the definitive RP (Additional file 1: Figure S1G, $\mathrm{H}$ and see [12]).

The transcription factor Foxj1 has been implicated in the formation of motile cilia. In the floor plate (FP), Foxj1 alters responsiveness of these ventral midline cells to Sonic hedgehog (Shh), prompting them to become refractory [24]. Evidence also points to its regulation by BMP signaling [37]. Foxj1 mRNA was only evident at the RP but not the NC stage, despite being transcribed continuously in the FP (Additional file 1: Figure S1I, J). Hairy1, a bHLH transcriptional repressor of the Hairy/ Hes family, revealed a similar profile of expression to that of Foxj1 (Additional file 1: Figure S1K, L). Thus, while Id1/2, LTK, Foxd3, Snail2, and Sox 9 are differential markers for the early NC stage, Foxj 1 and Hairy1 differentially map to the RP stage. Notably, Bmp4, Gdf7, $W n t 1, M a f B$, and $L m x 1 a / 1 b$ are continuously expressed throughout NT development, even if classically defined as RP markers (Fig. 2a, c; Additional file 1: Figure S1M-R; and see $[4,14,20,21])$. Taken together, the above cellular and molecular traits differentially define two discrete phases in dorsal NT development: NC and RP.

\section{BMP signaling is transient in the dorsal midline of the NT The definitive $R P$ cells are refractory to BMP signaling}

Next, we began addressing the mechanisms responsible for the transition between these stages. During patterning of the nervous system, the dorsal NT produces and secretes proteins of the BMP family that at early stages control the emigration of $\mathrm{NC}[16,17,25,38]$ and later induce, in a non-cell autonomous manner, the specification and differentiation of spinal interneurons [39, 40]. These activities of BMP prompted us to examine its involvement in the transition between $\mathrm{NC}$ and $\mathrm{RP}$ stages during dorsal NT development. 

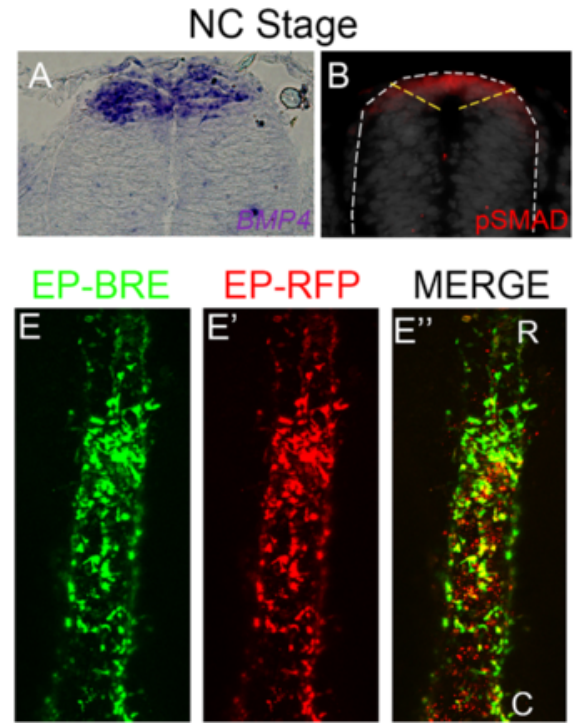
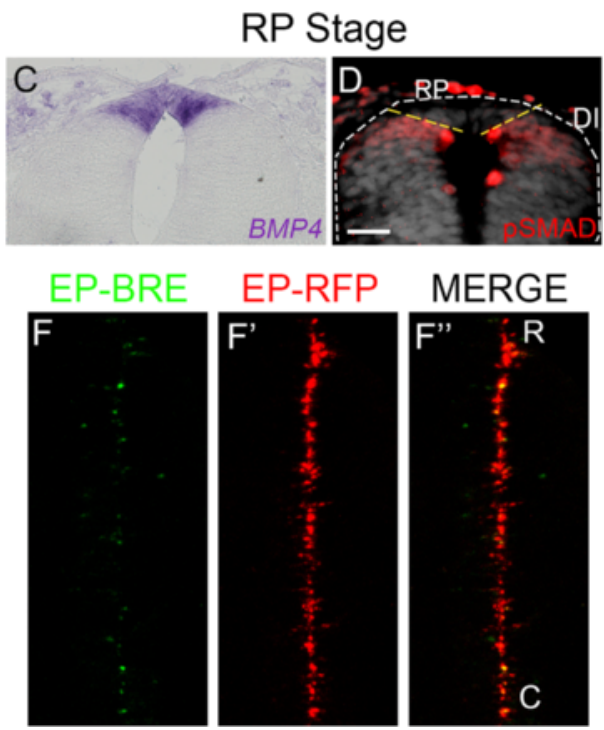

Fig. 2 The roof plate (RP) loses responsiveness to BMP signaling. a, $\mathbf{c}$ In situ hybridization for Bmp4 at the neural crest (NC) and RP stages. b, d Antibody staining for pSMAD1,5,8. Note positive signaling in the dorsal neural tube (NT) at the NC stage (delimited by dotted yellow lines, see "Methods" for definition). In contrast, the definitive RP (dorsal to the dotted yellow lines, see "Methods" for definition) lacks a pSMAD signal yet the domain ventral to the yellow lines containing dorsal interneurons is positive. e, $\mathbf{f}$ Dorsal views of whole mounted embryo fragments following dorsally directed electroporations (EP) of BMP-responsive element (BRE)-GFP along with control RFP to monitor electroporation efficiency. Note positive and negative BMP reporter signaling at NC and RP stages, respectively. Embryos were electroporated at either E2 (e-e') or E3 (f-f') and analyzed $16 \mathrm{~h}$ later. White dashed lines delineate the NT in $\mathbf{b}$ and $\mathbf{d}$. C caudal, D/ dorsal interneurons, $R$ rostral. Bar in $\mathbf{a}=40 \mu \mathrm{M}$; b, $\mathbf{d}=80 \mu \mathrm{M} ; \mathbf{c}=50 \mu \mathrm{M}$

In agreement with previous findings [4, 12], we observed that expression of Bmp4 transcripts was evident in the dorsal NT at both NC and RP stages (Fig. 2a, c). BMP activity was evidenced with anti-Phospho-Smad 1-5-8 (pSMAD) [41]. In contrast to Bmp4 transcription, pSMAD staining was positive in the dorsal NT only at the NC stage (Fig. 2b). At the RP stage, only dorsal interneurons, located ventral to the definitive RP, were pSMAD+, while the midline $R P$ domain was negative (Fig. 2d).

To further examine this stage-dependent difference in BMP sensitivity, we took advantage of a genetic reporter for BMP activity, consisting of a BMP responsive element (BRE) that drives expression of GFP [8, 18]. Focal electroporations of BRE-GFP together with RFP to control for transfection efficiency were directed to the dorsal NT at E2 (17-20 ss) or E3 (35 ss). Dorsal views of transfected neural primordia revealed many RFP+ cells at the NC stage exhibiting a BRE:GFP signal (Fig. 2e-e"), whereas only a few RFP+ cells approaching the RP stage were BRE:GFP+ (Fig. 2f-f") when examined $16 \mathrm{~h}$ after electroporation. Hence, while both NC and RP cells produce and secrete BMP proteins, only progenitors at the NC stage are responsive. The definitive RP loses competence to respond to the BMP ligand, while continuing to provide BMP to the ventrally localized interneurons and/or their progenitors.

\section{Prospective RP cells are initially responsive to $B M P$}

In a previous study [12] we demonstrated that preceding the onset of NC delamination, RP progenitors cells are located ventral to the presumptive $\mathrm{NC}$ pool, and that they relocate dorsally upon NC emigration. In addition, we found that, initially, prospective RP progenitors are part of the Foxd3+ lineage, but downregulate its expression during dorsal relocation [13]. We then hypothesized that RP progenitors are initially responsive to BMP signaling, given that all dorsal NT cells appear to be sensitive to BMP signaling at the NC stage (Fig. 2b, e). To test this notion, we transfected BRE-GFP to the flank of 22 ss (HH14)-stage embryos corresponding to the early NC stage. Embryos were re-incubated for an additional 24 or $36 \mathrm{~h}$ until reaching the RP stage. As predicted, the midline RP domain was BRE-GFP+ (Fig. 3a-a", b-b"). Because the RP is already insensitive to BMP by the time of fixation, (e.g., BRE-GFP-, see Fig. 2), the GFP signal observed in the dorsal NT at E3-E3.5 must result from an accumulation of the GFP protein following the early electroporation. Because GFP has a half-life of about 48 $\mathrm{h}$, it is possible to trace cells after the BMP signal has been turned off [42].

To control for this dynamic behavior, we performed similar electroporations a few hours later, in embryos aged 28-30 ss (HH16-17), still well within the NC stage 

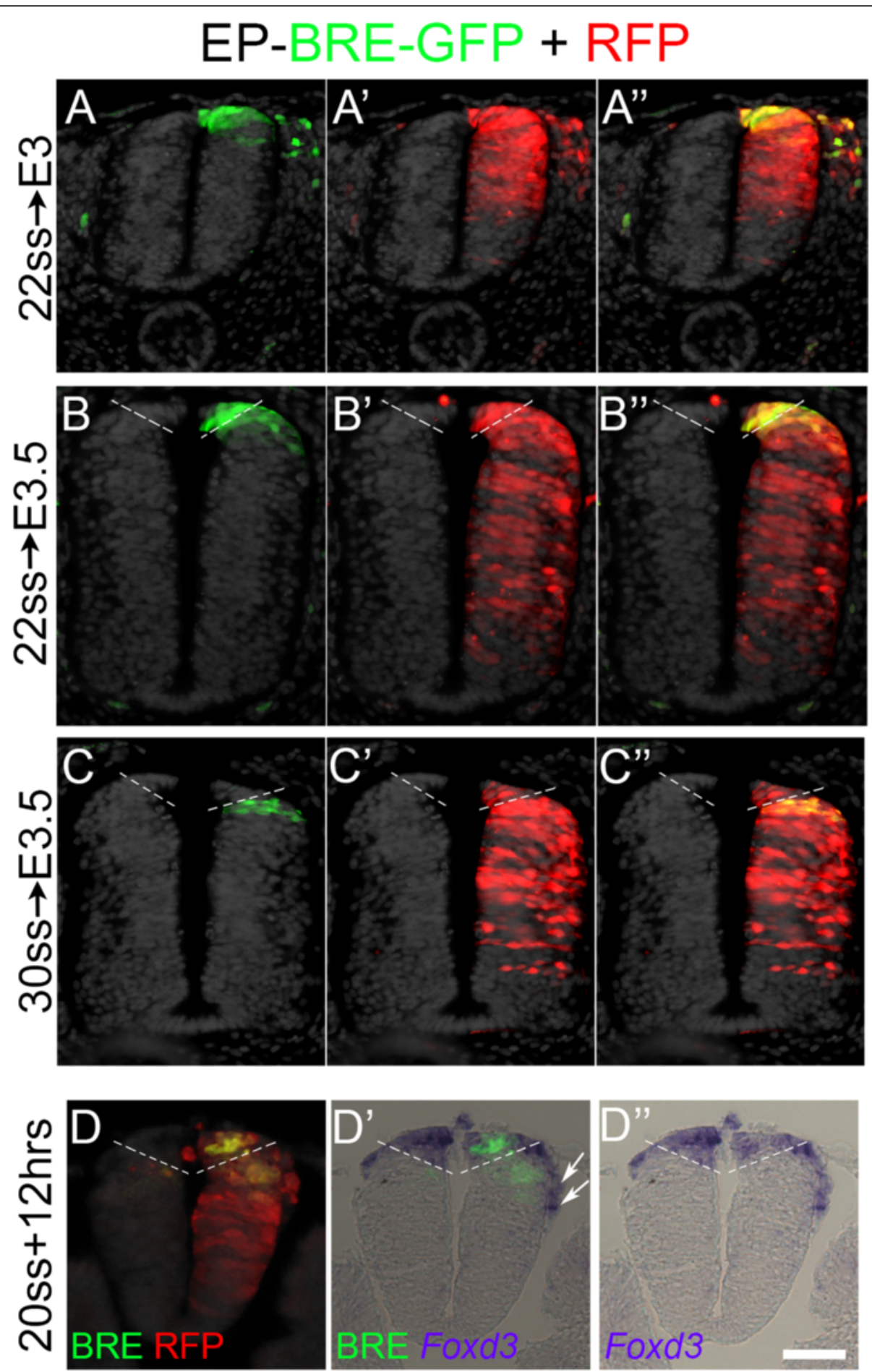

Fig. 3 Dynamics of BMP responsive element (BRE) in the dorsal neural tube (NT). a-c Hemi-NT electoporations of BRE-GFP along with control RFP to trace electroporation efficiency. Embryos were electroporated and analyzed at designated times. Note presence of positive BRE-GFP signal in RFP+ cells in the early electroporations $\left(\mathbf{a}-\mathbf{b}^{\prime \prime}\right)$. When electroporated later $\left(\mathbf{c}-\mathbf{c}^{\prime \prime}\right)$, the BRE-GFP signal is absent in the definitive roof plate (RP) but positive more ventrally. Dashed lines in $\mathbf{b}$ and $\mathbf{c}$ delimit the definitive RP (see "Methods"). $\mathbf{d}-\mathbf{d}$ " In situ hybridization for Foxd3 transcripts, showing co-localization with the BRE-GFP signal in the dorsal NT. Dashed lines mark the ventral limit of the Foxd3-positive domain. Note also the presence of Foxd3+ neural crest cells migrating dorso-ventrally outside the neuroepithelium (arrows). In addition, the BRE-GFP signal also extends further ventrally into a Foxd3-negative region. Bar for $\mathbf{a}-\mathbf{a} \mathbf{a}^{\prime \prime}=50 \mu \mathrm{M} ; \mathbf{b}-\mathbf{d}^{\prime \prime}=40 \mu \mathrm{M}$ 
$[12,43]$. Under these conditions, the RP was largely negative for the BRE-GFP signal (Fig. 3c-c"). In this time frame, prospective RP cells are either unable to accumulate enough GFP before losing their capacity to respond to BMP, or they have already lost BMP sensitivity. These results reveal a similar temporal dynamic to that of RP progenitors, which only transiently exhibited Foxd3 reporter activity [13]. For this reason, we analyzed embryos that were injected at an early stage with BREGFP and then in situ hybridized for Foxd3. The Foxd3+ signal was included within the dorsal domain of BREGFP+ expression, encompassing at this stage both NC and RP precursors [13]. In addition, the BRE-GFP signal extended further ventrally into a Foxd3- region, likely comprising dorsal interneuron progenitors (Fig. 3d-d"). Together, these results suggest that prospective RP progenitors initially respond to BMP and then lose sensitivity upon relocation to their definitive dorsal midline position.

Because BMP ligands are continuously produced by dorsal NT cells, we hypothesized that the development of RP insensitivity to BMP may be accounted for by a timely downregulation of BMP receptors. In situ hybridization revealed that while expression of Bmpr1b (Alk6) transcripts is apparent in the dorsal NT at both $\mathrm{NC}$ and RP stages, that of Bmprla (Alk3) is positive at the NC stage but downregulated at the RP stage (Additional file 2: Figure S2). This indicates that the downregulation of specific BMP receptors is part of a mechanism responsible for the loss of competence of nascent RP cells to respond to BMP.

\section{The dynamics of BMP signaling vis-à-vis RP progenitors}

Our results suggest that RP progenitors will respond to BMP signaling until they arrive at their dorsal midline position. Notably, because of their initial ventral localization with respect to the $\mathrm{NC}$ and their prolonged ventro-dorsal relocation, RP progenitors are likely subjected to BMP for longer than the NC cells, which progressively exit the NT. It was previously suggested that extended exposure to morphogens such as BMP and Shh is equivalent to generating a higher level of signaling required for the specification of distinct dorsal [18] and ventral [44] neuronal subsets, respectively. In such a case, exposing early neural progenitors to high BMP signaling should prematurely induce RP at the expense of NC. To test this possibility, a constitutively active (ca) version of Alk3 was used. In all cases $(\mathrm{N}=6)$, when electroporated into hemi-NTs at 18-20 ss and fixed a day later, an ectopic pSMAD signal was detected in transfected cells. This contrasted with an observed pSMAD expression restricted to the dorsal domain under control conditions $(\mathrm{N}=4)$ (Additional file 3: Figure S3).

Next, we examined the effects of caAlk3 on NC EMT, G1/S transition, and gene expression. Electroporations were directed ventro-dorsally, transfecting either the dorsal quadrant of hemi-NTs or their dorsal domain on both sides of the midline. Consistent with our hypothesis, NC EMT was dramatically inhibited upon transfection of caAlk3 $(\mathrm{N}=7)$ when compared to controls $(\mathrm{N}=5$, Fig. 4a, b), as was the extent of BrdU incorporation into the nuclei of transfected progenitors when challenged for a prolonged pulse of $2 \mathrm{~h}(\mathrm{~N}=7$ and 6 , respectively, $P<0.005$, Fig. 4c-e). In addition, by $12 \mathrm{~h}$ Foxd3 mRNA was downregulated in the caAlk3-electroporated progenitors $(\mathrm{N}=5$, Fig. $4 \mathrm{~g}$, g' arrow) compared to control GFP $\left(\mathrm{N}=3\right.$, Fig. 4f, $\left.\mathrm{f}^{\prime}\right)$, confirming that $\mathrm{NC}$ production was inhibited. Reciprocally, expression of the RP marker Hairy1 (Additional file 1: Figure S1K, L) was upregulated as early as $9 \mathrm{~h}$ post-transfection to levels similar to those apparent in the $\mathrm{FP}(\mathrm{N}=8$, Fig. 4i, i' arrow) whereas control GFP had no effect $(\mathrm{N}=4$, Fig. 4h, h').

Whereas NC proliferation and Foxd3 expression are inhibited by high BMP signaling, our previous results showed that inhibiting endogenous BMP activity by noggin at the early $\mathrm{NC}$ stage also attenuates $\mathrm{NC}$ proliferation, Foxd3 expression, and NC EMT [16, 45, 46]. Here, we further confirm and extend these data by showing that treatment with noggin, Smad6 (a negative effector of BMP signaling [47]), or a dominant negative form of $\mathrm{BMP}$ receptor (dnBMPR) [48] all caused a significant reduction of BrdU incorporation in premigratory NC cells $16 \mathrm{~h}$ after electroporation when compared to control GFP-treated embryos $(\mathrm{N}=4$ embryos per treatment, $P<$ 0.05, Additional file 4: Figure S4). Hence, both early inhibition as and high levels of BMP signaling compromise NC production. However, abrogation of endogenous BMP signaling did not promote a premature upregulation of Hairy1 expression ( $\mathrm{N}=5$, Additional file 4: Figure S4F), in contrast to the premature appearance of Hairy1 transcripts observed upon caAlk3 treatment. In addition, no Hairy 1 mRNA was apparent at the RP stage in embryos treated with either dnBMPR or noggin when compared to controls $(\mathrm{N}=4$ for each treatment, Additional file 5: Figure S5). Together, these results suggest that BMP signaling is both necessary as well as sufficient for expression of Hairy1 in RP and further indicate that development of RP properties requires high BMP signaling.

\section{Dynamics and function of Hes/Hairy1 in the dorsal NT Hes 1/Hairy 1 activity is restricted to the RP stage}

Hes1/Hairy1 is a candidate gene possibly involved in the transition between NC and RP stages because it is prematurely transcribed upon exposure of dorsal NT cells to high BMP signaling levels (Fig. 4i). Furthermore, Hairy1 mRNA is expressed at the RP but not the $\mathrm{NC}$ stage (Additional file 1: Figure S1). To further evaluate its involvement in this transition, we implemented the 


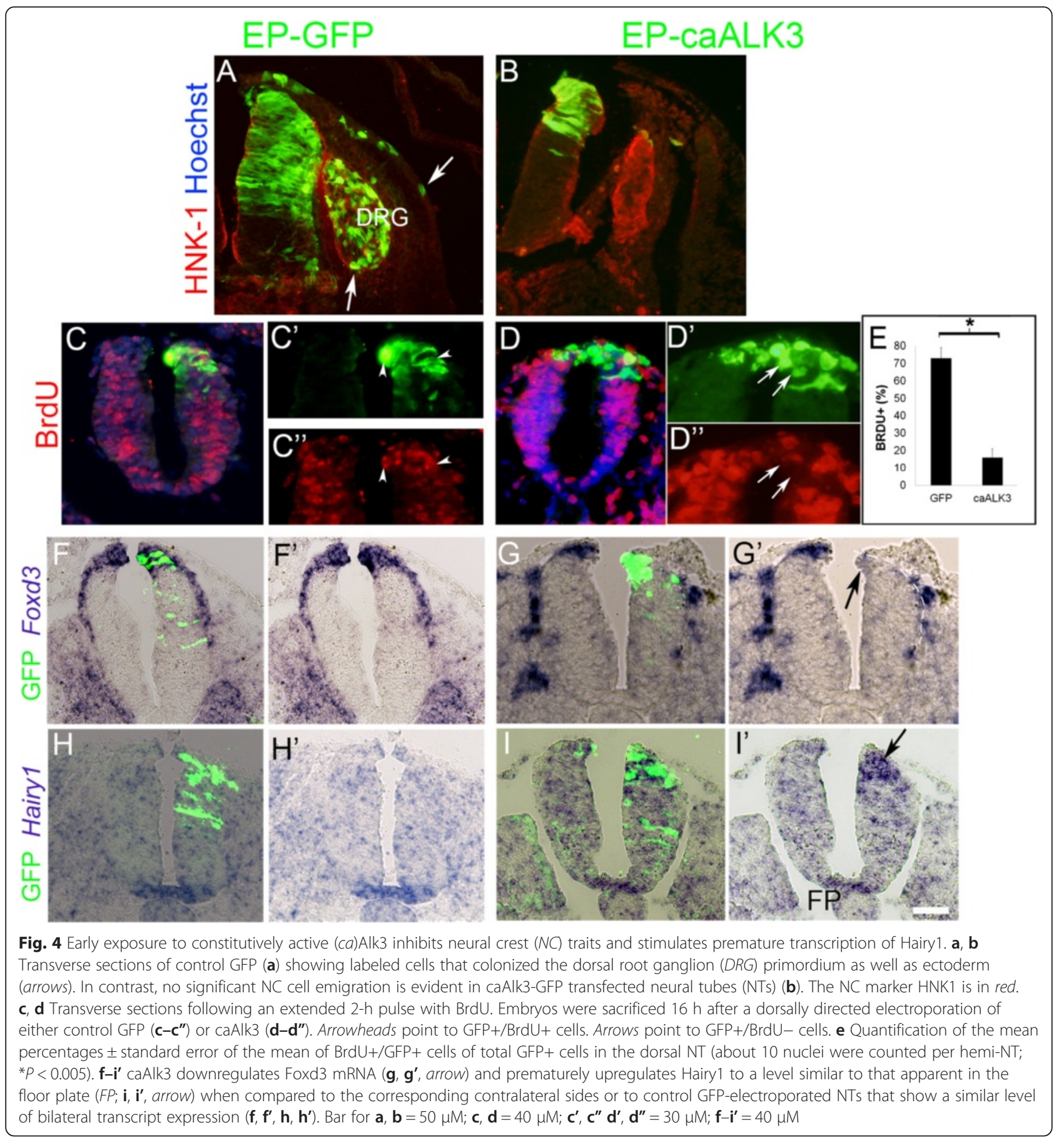

mouse Hes1 promoter, homologous to chick Hairy1 [49], that drives expression of a reporter GFP cassette [50]. Co-electroporation of the Hes1/GFP promoter and control RFP at the NC stage (17-18 ss) showed no GFP signal in the RFP+ progenitors when examined $10 \mathrm{~h}$ later $\left(\mathrm{N}=6\right.$, Fig. $\left.5 \mathrm{a}, \mathrm{a}^{\prime}\right)$. In contrast, the RFP+ RP cells were GFP+ when co-electroporation was performed at 40 ss $(\mathrm{N}=5$, Fig. 5b, b'). Thus, consistent with its mRNA expression pattern, Hes1 is specifically active in the RP.

\section{Hes1/Hairy1 inhibits NC delamination and cell-cycle progression}

Next, we examined whether misexpression of Hes/ Hairy1 at the NC stage may adversely affect NC behavior. We observed no delamination either 1 or 2 days 


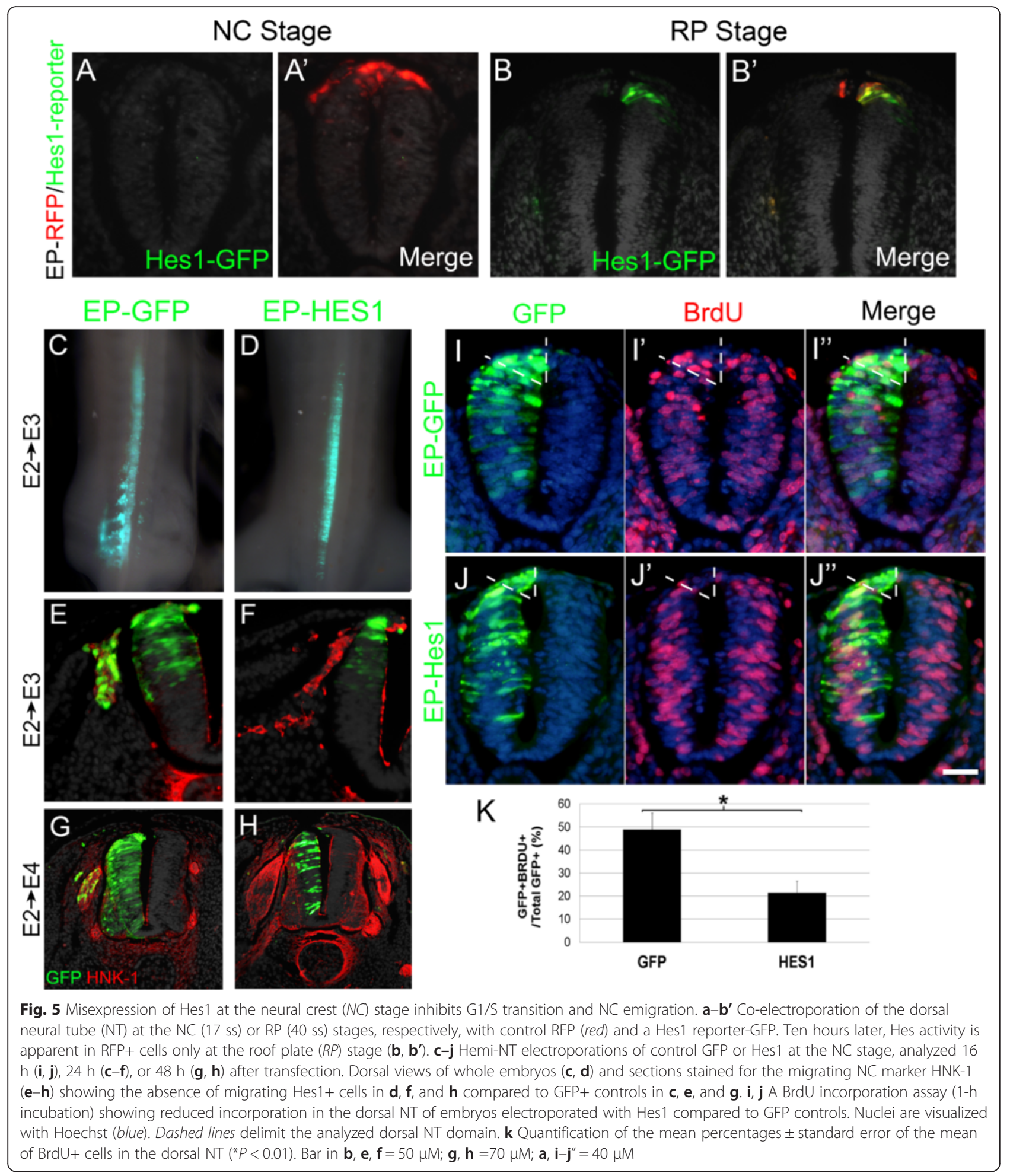

after electroporation of Hes1, the mouse Hairy1 homologue (Fig. 5d, f, h), when compared to control GFP-electroporated embryos (Fig. 5c, e, g). Next, we found that Hes1 misexpression promotes premature cell-cycle exit in the electroporated cells, evidenced by the inhibition of BrdU incorporation when compared to GFP controls $(\mathrm{N}=5$ for each treatment, Fig. 5i-k, $P<0.01)$. 
Hes 1/Hairy1 attenuates BMP responsiveness in the dorsal NT and downregulates Alk3 mRNA expression

The preceding results indicate that Hes1 plays a role in ending NC production. This could be accounted for by Hes1 exerting a negative feedback on BMP signaling or on genes acting downstream of BMP. Such a Hes1dependent loss of endogenous BMP activity in dorsal NT cells might explain the loss of responsiveness to BMP observed upon transition into the RP stage, which causes a concomitant exit from the cell cycle and the end of NC EMT.

To examine this possibility, Hes1 was electroporated at the NC stage, and pSMAD expression analyzed $20 \mathrm{~h}$ later. In control GFP-transfected tubes, pSMAD staining was similarly expressed on both sides of the dorsal NT (Fig. 6a-a"). In contrast, NTs transfected with Hes1/ Hairy1 displayed a significant reduction in pSMAD staining in the transfected hemi-NTs (Fig. 6b-b", arrow in b').

Next, we asked whether the Hes1-dependent loss of BMP activity can be explained by a downregulation of Alk3 receptors, whose expression is normally lost in the transition between NC and RP stages (see Additional file 2: Figure S2). Fifteen hours after the co-electroporation of Hes1 and GFP-encoding plasmids at the NC stage, we observed a premature reduction of $A l k 3$ mRNA signal in the
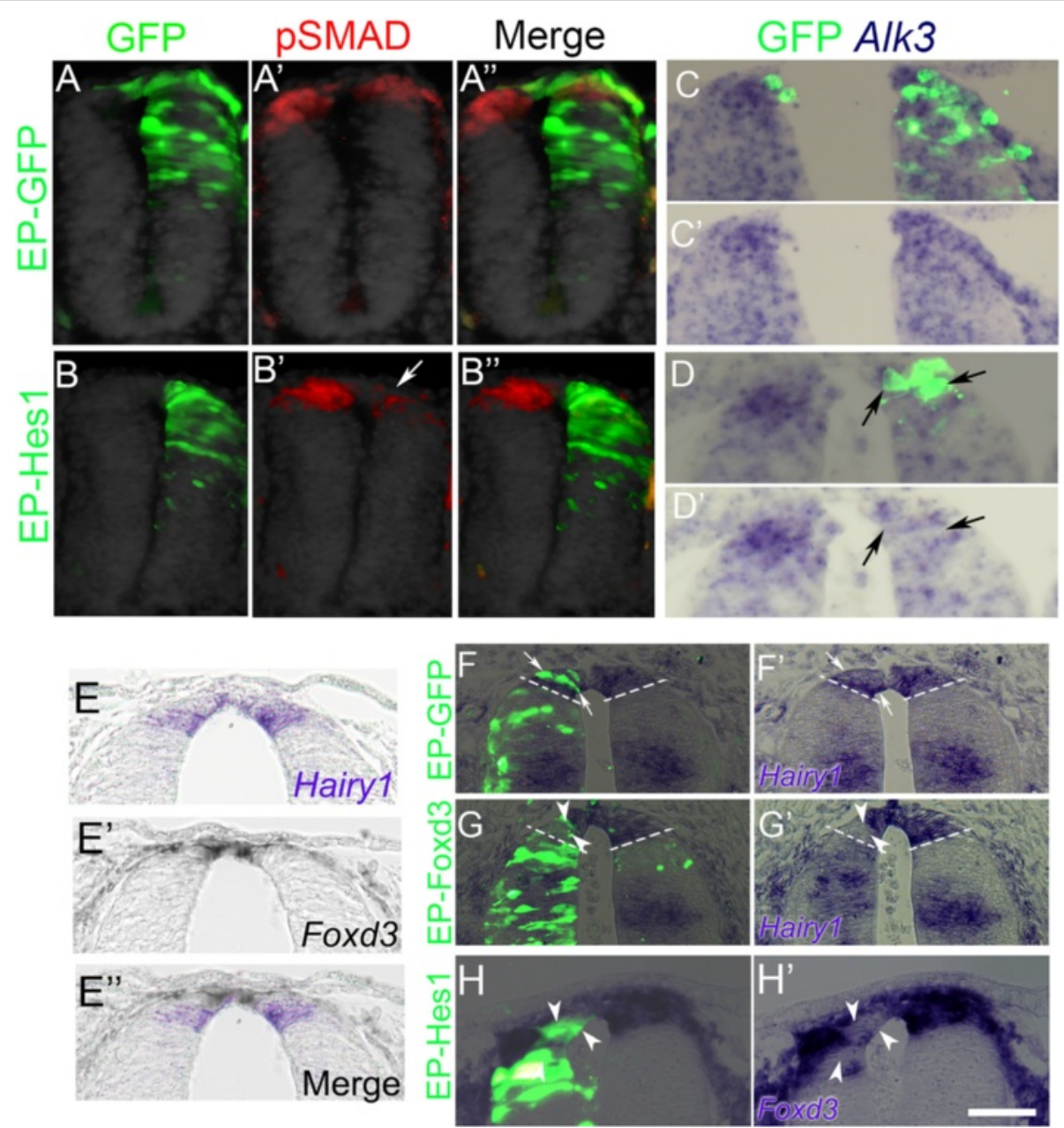

Fig. 6 The relationship between Hes1/Hairy1, BMP signaling, and downstream Foxd3 expression. a-b" Embryos were electroporated with either

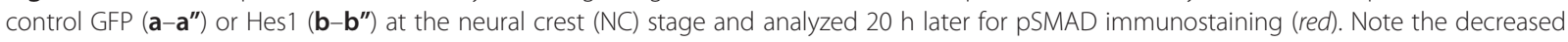
pSMAD signal in the treated side at $\mathbf{b}^{\prime}$ (arrow) compared to the untransfected side and to control GFP. c- $\mathbf{d}^{\prime}$ Embryos were electroporated with either control GFP $\left(\mathbf{c}, \mathbf{c}^{\prime}\right)$ or Hes1/GFP $\left(\mathbf{d}, \mathbf{d}^{\prime}\right)$ at the NC stage and analyzed $15 \mathrm{~h}$ later for Alk3 mRNA expression. Note the reduction in the Alk3 signal in the transfected (green) side of the Hes-treated hemi-neural tube (NT) (white arrow). e-e" Mutually exclusive spatial expression domains of Foxd3 and Hairy1. In situ hybridization on adjacent sections of the same embryo performed at 35 ss. Note that at this particular stage, Hairy 1 expression (blue) is ventral to the Foxd3+ (black) domain. $\mathbf{f}, \mathbf{g}^{\prime}$ Foxd3 and Hairy1 inhibit each other's expression. Electroporation of Foxd3 at E2.5 cell autonomously inhibits Hairy 1 expression in the dorsal NT $16 \mathrm{~h}$ later (g, $\mathbf{g}^{\prime}$; arrowheads), compared to the contralateral side and to GFPelectroporated controls ( $\mathbf{f}_{\mathbf{f}} \mathbf{f}^{\prime}$; arrows). $\mathbf{h}, \mathbf{h}^{\prime}$ Electroporation of Hes1 at the NC stage inhibits Foxd3 transcription compared to the contralateral side. Arrowheads point to GFP+/Foxd3- cells. For control see Fig. 4f. Bar for $\mathbf{a}-\mathbf{b}^{\prime \prime}, \mathbf{f}-\mathbf{g}^{\prime}=60 \mu \mathrm{M} ; \mathbf{c}-\mathbf{e}^{\mathbf{\prime}}, \mathbf{h}, \mathbf{h}^{\prime}=50 \mu \mathrm{M}$ 
transfected hemi-NTs $(N=9$, Fig. 6d, d') when compared to control GFP alone ( $\mathrm{N}=6$, Fig. $\left.6 \mathrm{c}, \mathrm{c}^{\prime}\right)$.

Thus, the onset of Hairy1 production in the nascent RP might be sufficient to inhibit both BMP receptor expression and BMP responsiveness, consequently abrogating BMP-dependent G1-S transition and cell delamination.

\section{A mutual cross-inhibition between Foxd3 and Hairy1}

The transcription factor Foxd3 is a BMP-dependent gene expressed from early stages onwards in the dorsal NT $[4,13,16,51]$. Consistently, Foxd3 is transcribed at the NC but not definitive RP stages (Additional file 1: Figure S1G, H) [12, 13]. Conversely, expression of Hes1/Hairy1 is largely reciprocal to that of Foxd3 (Additional file 1: Figure S1K, L and Fig. 5). To further examine their differential expression, we performed in situ hybridization on embryos aged 33-36 ss (HH1718), an intermediate stage corresponding to the dorsal restriction of the Foxd3-positive domain [12] and the onset of Hairy1 transcription (e.g., the transition between NC and RP stages). In these embryos, Foxd3 and Hairy1 were not co-expressed in the same cells. Whereas Foxd3 mRNA was already restricted to a narrow strip of progenitors located in the dorsal midline, Hairy1 expression was apparent immediately ventral to the Foxd3-positive domain, presumably corresponding to prospective RP progenitors (Fig. 6e, e'). These results further strengthen the dynamic behavior of dorsal NT precursors where RP progenitors are situated ventral to the premigratory NC prior to arriving at their definitive position [12].

Based on their reciprocal expression patterns, we hypothesize that Foxd3 and Hes1/Hairy1 may stand in a mutually repressive interaction. Misexpression of Foxd3 close to the end of the NC stage inhibited in a cellautonomous manner the transcription of Hairy1 when analyzed at the RP stage $(\mathrm{N}=5$, arrowheads in Fig. $\left.6 \mathrm{~g}, \mathrm{~g}^{\prime}\right)$ in comparison to GFP controls $(\mathrm{N}=5$, arrows in Fig. 6f, $\left.\mathrm{f}^{\prime}\right)$. In addition, misexpression of Hes1 at the NC stage repressed Foxd3 mRNA in the dorsal NT when compared to the untreated side and to control GFP ( $N=6$, arrowheads in Fig. $6 \mathrm{~h}$, h' and see Fig. 4f, f' for control GFP).

These results suggest that Foxd3 and Hairy1 negatively regulate each other, thus maintaining both spatial and temporal separation between $\mathrm{NC}$ and RP properties in the dorsal neuroepithelium.

\section{Discussion}

During the development of the CNS, neuroepithelial cells gain distinct identities and give rise to numerous cell types. In the region of the NT destined to become the spinal cord, the acquisition of distinct fates is coordinated by the RP and FP, organizing centers secreting diffusible instructive signals. But how do the organizing centers themselves form and gain their proper fates? While significant work has been invested in understanding FP development [52], very little is known about the formation of the RP. This is further complicated by the highly dynamic behavior of this region, which is first populated by $\mathrm{NC}$ progenitors and only after their exit from the NT becomes the RP, a definitive group of CNS cells.

Here, we have begun to unravel the mechanism that accounts for the transition between NC and RP stages. First, we have identified a number of cellular and molecular traits that characterize each stage. Second, we report that although initially responsive to BMP, RP progenitors lose competence to respond to the ligand upon transition to their definitive location. In parallel, RP cells upregulate Hairy1, which is likely to confer the observed insensitivity to BMP signaling, despite the fact that they continuously synthesize the ligand. Consequently, NC production and emigration end, presumably due to a cell-cycle arrest induced by Hairy1 via downregulation of BMP receptors and consequent BMP signaling. In addition, Foxd3 and Hairy1 not only have non-overlapping temporal expression patterns, but also inhibit each other's transcription. Together, this constitutes a negative regulatory loop at the level of, and downstream from, BMP signaling that controls the sequential production of NC followed by RP (Fig. 7).

Classically, the term RP is generically used to define the dorsal domain of the NT, in particular with regard to its morphogen-secreting capacity and function in patterning of dorsal interneurons [14, 22, 39]. However, compounds that are usually considered as RP markers-such as morphogens like BMPs and Wnts, and transcription factors like MafB and Lmxla/b-are produced throughout dorsal NT development, encompassing the early NC period $[4,5]$. Clearly, the dorsal NT differs significantly between the stages both in terms of cell fates and cellular behaviors, demonstrating the need for a more exact definition.

Our previous experiments [12, 13] showed that progenitors of the definitive RP are initially located ventral to the prospective NC. Initially, these cells are molecularly indistinguishable from presumptive $\mathrm{NC}$ because they also express Foxd3, as evidenced by the use of a Foxd3 reporter [13], and are still responsive to BMP, as revealed here by lineage analysis with a BRE-GFP reporter. Upon NC emigration, prospective RP cells relocate dorsally toward their definitive midline position, and during this time they become refractory to BMP, downregulate $A l k 3$, and cease to express Foxd 3 and the direct BMP target genes Id2/3. The initiation of Hairy1 expression must be associated with these events, because it is initially evident in a band of cells localized ventrally to the Foxd3+ domain; later, when BMP responsiveness 


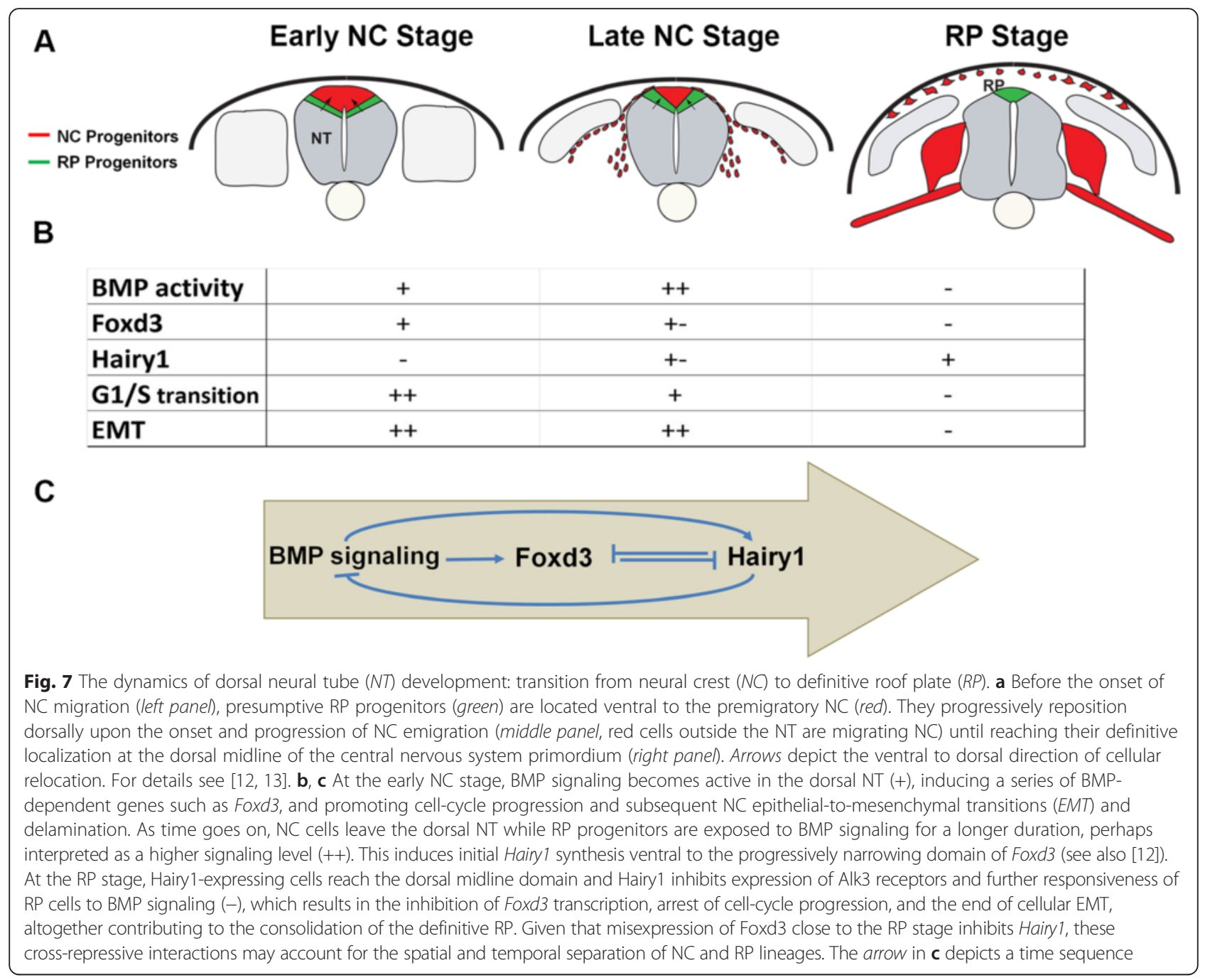

and Foxd 3 transcription are lost, Hairy1 expression and activity are evident in the most dorsal NT domain. This correlates in time with the completion of $\mathrm{NC}$ emigration.

This view would suggest that the functions of BMP and Wnt signaling differ between the NC and RP stages. Studies so far have addressed the role of these signaling molecules on various aspects of interneuron development yet misexpressed the ligands starting at the NC stages and analyzed the outcome 2 days later, thus encompassing both phases $[8,18,53]$. Clearly, limiting the loss of either BMP or Wnt function to the NC phase inhibits G1/S transition and the consequent EMT of these precursors by activating a molecular network that includes N-cadherin processing and Rho-GTPase activity $[9,17,38,54]$. However, it remains unclear whether such an early and time-limited inhibition suffices for preventing or altering interneuron development, and whether the latter cells require a continuous supply of the factors or, alternatively, whether a late supply by the definitive RP suffices given that interneuron progenitors still exhibit BMP responsiveness at this stage (Fig. 2). Another possibility would be that signals emanating from the young dorsal NT are important for interneuron specification and then differentiation $[8,18]$, whereas those from the definitive RP are necessary for axonal outgrowth and/or guidance. Taken together, we sustain that the use of the term RP to describe the structure emerging upon NT closure is inappropriate and propose instead to use the term RP only when NC delamination has ended, and the full segregation between CNS and PNS lineages is evident.

Members of the Hes/Hairy family of transcriptional repressors were found to be constitutively expressed in neural boundary domains, like the RP and FP of the spinal cord [22]. Persistent and high Hes1 expression levels repress both proneural gene transcription and cell proliferation in boundary regions within the nervous system $[22,55]$, whereas in the absence of Hes, Mash1 and other proneural genes are ectopically expressed in these domains $[22,56]$. In addition, an oscillatory expression 
of Hes genes has been documented in developing neuroblasts. This oscillatory behavior was critical to maintain progenitor cells in an undifferentiated state while inhibiting neuronal differentiation [57]. Furthermore, Hes/ Hairy genes were found to play a role in regulating cellcycle progression [58]. Whereas low levels of Hes1 promote cell proliferation by downregulating p21 and p27 [59], persistent and high levels of Hes1 were shown to inhibit the cell cycle [22, 60]. Consistently, as shown here, the advent of the RP is associated with cell-cycle exit and the onset of Hairy1 transcription. Moreover, Hes misexpression at the NC stage inhibited G1/S transition and $\mathrm{NC}$ production, suggesting that Hairy1 is functionally involved in ending $\mathrm{NC}$ production and $\mathrm{RP}$ formation.

An open question is what are the mechanisms that trigger Hes1/Hairy1 expression at the RP stage. Because both NC and RP precursors lie within a domain of high BMP signaling that expresses $M s x 1 / 2$ [18], we speculate that it is the comparably extended exposure of the latter to BMP that induces RP properties including Hairy1; this might occur by translating the longer duration of signal into a higher level of activity $[44,61]$ as suggested by our caAlk3 experiments. In addition, given that Foxd3 represses transcription of Hairy1 (our data) and suppresses interneuron development [62], it is logical to assume that Hairy1 transcription can only be stabilized in RP cells when Foxd3 itself is repressed or when Foxd3+ NC progenitors exit the NT. Our data moreover suggest that Hairy1 itself contributes to the inhibition of Foxd3 mRNA, either directly or indirectly by abrogating the responsiveness of dorsal NT cells to BMP signaling, the latter being a positive regulator of Foxd3 transcription [4, 16, 51]. Indeed, high levels of BMP activity (and/or longer signal duration) might be sufficient for inducing Hairy1 and repressing Foxd3 transcription in the dorsal NT, thus generating a feedback network of transcriptional interactions that consolidate RP formation (see Fig. 7).

In addition to expressing Hes/Hairy, FP and RP share similar roles because these boundary domains act as organizing centers that secrete morphogens to pattern neuronal differentiation in adjacent cells. Previously, high concentrations of the Shh morphogen, which is secreted by the notochord, were shown to be needed in both mouse and avian embryos to specify the ventral midline domain as FP. Later, to complete FP differentiation, these cells become refractory to the ligand $[24,52]$. In addition, Foxj1 is upregulated in FP cells in association with the reduction in Shh responsiveness, and Foxj1 alters the sensitivity of cells to Shh signaling, presumably by inducing long, motile cilia [24]. By analogy to the FP, we show here that upon NT closure and during the NC stage, dorsal progenitors respond to $\mathrm{BMP}$, but while transiting to the dorsal midline they downregulate Alk3 receptors, stop responding to BMP, and similarly upregulate Foxj1, regaining apically organized Arl13b + cilia and apico-basal polarity. Thus, in spite of the delayed development of the RP when compared to the FP [10], there are notable similarities between the specification of both ventral and dorsal organizing centers. Further investigation is needed to substantiate the mechanistic similarities between FP and RP development by examining, for instance, whether Foxj1 in the RP similarly alters BMP responsiveness and cilia morphology and function.

\section{Conclusions}

By initially characterizing a set of positive and negative activities involving regulated BMP signaling and Hes/Foxd3 interactions, our results provide novel insights into the dynamic events leading to the transition from the $\mathrm{NC}$ to the RP phase of NT development. Four main processes are noteworthy: first, our finding that RP progenitors initially respond to BMP yet lose competence upon relocation to their definitive dorsal midline position in the NT, where they finally consolidate their identity; second, that BMP signaling induces Hes transcription, which in turn downregulates BMP responsiveness, likely through modulation of Alk3 receptor transcription; third, that downstream of BMP, a cross-repressive interaction between Foxd3 (an NC marker) and Hairy1 (an RP marker) accounts for the temporal and spatial segregation of both lineages; and fourth, that in spite of being refractory, the definitive RP continues producing BMP, which is likely to act upon dorsal interneurons. The precise time-dependent activities of $\mathrm{BMP}$ emanating from the early (NC stage) versus late (RP stage) dorsal NT remain to be defined. These multiple roles of BMP signaling indicate that its function is context dependent and dictated by the regulatory state and competence of the target cells. We also notice that RP ontogeny bears significant resemblance to the development of the FP, initiated by Shh signaling in the ventral NT, both in terms of signal duration/intensity followed by refractoriness. Future research should focus on unveiling additional genes and interactions that comprise the differential molecular networks underlying the sequential functions of BMP on NC, RP, and interneuron development.

\section{Methods \\ Embryos}

Chick (Gallus gallus) and quail (Coturnix japonica) eggs were obtained from commercial sources (Moshav Orot and Moshav Mata, respectively). Experiments were conducted at the flank level of the axis (20-25 ss).

\section{Expression vectors and electroporation}

Expression vectors were: pCAGGS-EGFP, pCAGGS-RFP [12], pBI-EGFP, Noggin [9], pCAB-Smad6, pCAB-dnBMPR 
[48], pBI-mHes1 (subcloned from [50]), pBI-cFoxd3 $[43,62]$, and pBI-caBMPR1a/Alk3 (from D. Schulte and subcloned into pBI). To trace Hairy1 activity, a $2.5 \mathrm{~kb}$ mouse Hes1 promoter driving expression of a GFP reporter (pHes1-promoter-GFP) was used (N. Jing [50]). The specificity of the Hes1 reporter was previously assessed by monitoring changes to Notch signaling [33]. To monitor BMP signaling, we implemented a BRE that drives expression of a GFP reporter plasmid. BRE-GFP contains two copies of two distinct and conserved elements of the binding sites for Smad4 upstream of a minimal thymidine kinase promoter (E. Marti; [8]). Specificity of the BMP reporter was previously verified by co-electroporation with pEFBOS-mBMP4 [33].

Both the pHes1 promoter and BRE-GFP plasmids were electroporated along with a control RFP-encoding vector, to monitor electroporation efficiency.

DNA $(2-5 \mathrm{mg} / \mathrm{ml})$ was microinjected into the lumen of the NT at the trunk level of the axis at specific stages as detailed for each experiment. For hemi-NT electroporations, $5 \mathrm{~mm}$ tungsten electrodes were placed on either side of the embryo. For discrete electroporations into the dorsal NT, a $5 \mathrm{~mm}$ tungsten electrode was inserted under the blastoderm and a fine, 1-2 mm long electrode placed over the dorsal NT. A square wave electroporator (BTX, San Diego, CA, USA) was used to deliver one to three pulses of current at $10-20 \mathrm{~V}$ for $10 \mathrm{~ms}$.

\section{Immunohistochemistry and in situ hybridization}

Antibodies against HNK1 (CD57, BD Biosciences, San Jose, CA, USA Cat\#559048, 1:500), Arl13b (from Tamara Caspary, 1:1000), ZO-1 (Thermo Fisher Scientific, Waltham, MA USA, cat\#402200, 1:100), Ncadherin (R\&D Systems, Minneapolis, MN, USA., cat\#BTA7, $5 \mu \mathrm{g} / \mathrm{ml}$ ), laminin (Sigma-Aldrich, St. St. Louis, MO, USA cat\#L9393,1:100), BrdU (G3G4, Developmental Studies Hybridoma bank, Iowa City, Iowa, USA 1:100), and phosphorylated Smad 1-5-8 (pSMAD, from Ed Laufer, 1:1000) were used as previously described [9]. Cell nuclei were visualized with Hoechst. In situ hybridization was performed on paraffin sections as described [25]. The following probes were employed: BMP4 [16], Foxd3 [62], Bmpr1a/Alk3, Bmpr1b/Alk6 [63], Hairy1 [49], Id2, Id3 [33], Foxj1 [64], MafB [65], Wnt1 [4], and Gdf7 (from A. Graham).

\section{Data analysis and statistics}

The dorsal NT domain at NC and RP stages was analyzed. At the NC stage, the expression domain of Foxd3 generally contains $8-14(9.57 \pm 1.18)$ nuclei, and the expression domain of MafB at the RP stage contains 10$15(13.5 \pm 0.94)$ nuclei per hemi-NT. Therefore, for cell counts and domain definition at either stage, the most dorsal 8-10 nuclei per hemi-NT were considered.
Five to twelve embryos were analyzed per experimental treatment. For BrdU incorporation measurements, cells in 5-20 sections per embryo were counted and expressed as percentage of $\mathrm{BrdU}+/$ total $\mathrm{GFP}+$ cells in the dorsal NT.

Images were photographed using a DP70 (Olympus, Japan) cooled charge-coupled device digital camera mounted on a BX51 microscope (Olympus, Japan). Confocal sections of whole-mount preparations encompassing their entire thickness were photographed using a Nikon Eclipse 90i microscope with a Plan Apo $10 \times / 0.45$ dry objective (Nikon, Japan) and a D-Eclipse c1 confocal system (Nikon, Japan) at $2.7 \mu \mathrm{m}$ increments through the z-axis. Images were z-stacked with EZ-C1 3.90 FreeViewer software. For figure preparation, images were exported into Photoshop CS6 (Adobe). If necessary, the levels of brightness and contrast were adjusted to the entire image and images were cropped without color correction adjustments or $\gamma$ adjustments. Final figures were prepared using Photoshop CS6.

Data were subjected to statistical analysis using the nonparametric Mann-Whitney and Kruskal-Wallis tests. All tests applied were two-tailed and a $P$-value $\leq 0.05$ was considered significant.

\section{Additional files}

Additional file 1: Figure S1. Stage-specific expression of selected dorsal NT markers. (A-H) In situ hybridization for Id2 (A, B), Id3 (C, D), LTK (E, F), and Foxd3 $(G, H)$ at NC and RP stages. Note selective expression at the NC stage and no expression in the definitive RP. Among additional expression patterns, both $/ d 2 / 3$ are transcribed ventrally to the RP, corresponding to dorsal interneurons (B, D). (I-L) Foxj1 and Hairy1 are transcribed in the definitive RP $(J, L)$ but not the dorsal NT at the NC stage $(I, K)$. Note that both are expressed in the floor plate. (M-R) Gdf7, Wnt1, and MafB are expressed at both stages in the dorsal NT. Bar for $A, C, E, G, I, K, M, O$, $\mathrm{Q}=40 \mu \mathrm{M} ; \mathrm{B}, \mathrm{D}, \mathrm{F}, \mathrm{H}, \mathrm{J}, \mathrm{L}, \mathrm{N}, \mathrm{P}, \mathrm{R}=70 \mu \mathrm{M}$. (JPG $1246 \mathrm{~kb}$ )

Additional file 2: Figure S2. Expression of BMP receptors in the NT. (A-E) In situ hybridization for Bmprla/Alk3 (A-C) and Bmprib/Alk6 (D, E) at $N C(A, B, D)$ and RP stages $(C, E)$. $A$ and $D$ depict an early $N C$ stage opposite epithelial somites, and B illustrates an advanced migratory stage. Dashed lines in $C$ and $E$ delimit the definitive RP. Bar for A, B, $\mathrm{D}=60 \mu \mathrm{M} ; \mathrm{C}, \mathrm{E}=100 \mu \mathrm{M}$. (JPG $186 \mathrm{~kb})$

Additional file 3: Figure S3. caBMPR1A/caAlk3 stimulates ectopic pSMAD activity. (A-A") Electroporation of control GFP (PBI-GFP) or caBMPR1a-GFP-PBI (B-B") followed by pSMAD immunostaining. Note in $A-A^{\prime \prime}$ that the PSMAD signal is restricted to the dorsal NT even if the transfection attains half of the NT length. In contrast, caBMPR1a/Alk3 induces ectopic PSMAD activity in transfected cells throughout the electroporated domain (B-B"). Bar =50 HM. (JPG 516 kb)

Additional file 4: Figure S4. Inhibition of BMP signaling abrogates G1/ $\mathrm{S}$ transition in the dorsal NT but does not stimulate premature Hairyl expression. (A-D) Transverse sections showing the NT following a $1 \mathrm{~h}$ pulse of BrdU. Embryos were sacrificed $16 \mathrm{~h}$ after electroporation of either control GFP $\left(A-A^{\prime \prime \prime}\right)$, Noggin $\left(B-B^{\prime \prime \prime}\right)$, Smad6 $\left(C-C^{\prime \prime \prime}\right)$, or dnBMPR (D$\left.\mathrm{D}^{\prime \prime \prime}\right)$. Arrowheads point to GFP+/BrdU+ cells. Arrows point to GFP+/BrdUcells. (E) Quantification of the mean percentages \pm standard error of the mean of BrdU+/GFP+ cells of total GFP+ cells in the dorsal NT (about 10 nuclei were counted per hemi-NT, $N=4$ embryos for each treatment; $\left.{ }^{*} P<0.05\right)$. Nuclei are visualized with Hoechst (blue). $\left(F, F^{\prime}\right)$ Misexpression of noggin-GFP (green) has no effect on the expression of Hairy 
transcripts. $\mathrm{F}^{\prime}$ is an overlay of noggin-GFP and Hairy 1 in situ hybridization. Bar $=40 \mu \mathrm{M}$. (JPG $700 \mathrm{~kb})$

Additional file 5: Figure S5. Inhibition of BMP signaling prevents Hairy 1 expression in the dorsal midline region of the NT at the RP stage. Electroporations at the 10-15 ss of control GFP $\left(A-A^{\prime \prime}\right)$, dnBMPR (B-B"), or noggin $\left(C-C^{\prime \prime}\right)$ followed by fixation at $45 \mathrm{ss}$. Note Hairy 1 expression in the GFP+ RP of the control NT and the presence of many GFP-labeled, NCderived cells in the DRG. In contrast, no Hairyl signal is apparent in the hemi-RP misexpressing dnBMPR-GFP (asterisk in $B^{\prime}$ ), or in the entire RP that received noggin/GFP (asterisk in $C^{\prime}$ ). Arrows in $A^{\prime}, B^{\prime}$, and $C^{\prime}$ point to the RP. As expected from the known effects of BMP signaling on NC delamination, few or no labeled NC-derived cells were observed in peripheral targets of these embryos. DRG dorsal root ganglion, FP floor plate, NT neural tube. Bar $=60 \mu \mathrm{M}$. (JPG $858 \mathrm{~kb})$

\section{Abbreviations}

bHLH: basic helix-loop-helix; BMP: bone morphogenetic protein; BMPR: bone morphogenetic protein receptor; BrdU: bromodeoxyuridine; BRE: BMPresponsive element; ca: constitutively active; CNS: central nervous system; DRG: dorsal root ganglion; E: embryonic day; EMT: epithelial-to-mesenchymal transition; EP: electroporation; FP: floor plate; GFP: green fluorescent protein; $\mathrm{HH}$ : Hamburger and Hamilton stage; LTK: leukocyte tyrosine kinase; NC: neural crest; NT: neural tube; PNS: peripheral nervous system; RFP: red fluorescent protein; RP: roof plate; Shh: Sonic hedgehog; ss: somite stage.

\section{Competing interests}

The authors declare that they have no competing interests.

\section{Authors' contributions}

EN and CK conceived the project. EN and OA performed most experiments and data analysis. NK analyzed the effect of Hes1 on Alk3 expression. SO analyzed Hes reporter activity at the NC and RP stages. DK contributed data to Figs 4 and S5. EN and CK wrote the manuscript. All authors read and approved the final manuscript.

\section{Acknowledgements}

We thank E. Marti and N. Jing for sharing BRE and Hes reporters, respectively. We are indebted to numerous colleagues for sharing reagents, Tallie Bdolach for assistance with statistics, and Joel Yisraeli and all members of our group for discussions and critical reading of the manuscript. This work was supported by a fellowship from the Safra Center for Neurosciences (ELSC) to $\mathrm{OA}$, by a post-doctoral fellowship from the Council for Higher Education (CHE) to DK, and by grants from the DFG (UN34/27-1) and the Israel Science Foundation (\#97/13) to CK.

\section{Author details}

'Department of Medical Neurobiology, IMRIC and ELSC, Hebrew University of Jerusalem-Hadassah Medical School, Jerusalem 9112102, PO Box 12272, Israel. ${ }^{2}$ Present Address: Department of Molecular Cell Biology, Weizmann Institute of Science, Rehovot, Israel. ${ }^{3}$ Present address: Department of Genetics, Washington University, St. Louis, MO, USA.

\section{Received: 9 February 2016 Accepted: 10 March 2016}

\section{Published online: 24 March 2016}

\section{References}

1. Le Douarin NM, Kalcheim C. The neural crest. 2nd ed. New York: Cambridge University Press; 1999.

2. Bronner ME. Formation and migration of neural crest cells in the vertebrate embryo. Histochem Cell Biol. 2012;138(2):179-86.

3. Kalcheim C. Mechanisms of early neural crest development: from cell specification to migration. Int Rev Cytol. 2000;200:143-96.

4. Krispin S, Nitzan E, Kalcheim C. The dorsal neural tube: a dynamic setting for cell fate decisions. Dev Neurobiol. 2010;70:796-812.

5. Chizhikov W, Millen KJ. Mechanisms of roof plate formation in the vertebrate CNS. Nat Rev Neurosci. 2004:5(10):808-12.

6. Timmer JR, Wang C, Niswander L. BMP signaling patterns the dorsal and intermediate neural tube via regulation of homeobox and helix-loop-helix transcription factors. Development. 2002;129(10):2459-72.
7. Zechner D, Muller T, Wende H, Walther I, Taketo MM, Crenshaw 3rd EB, et al. Bmp and Wnt/beta-catenin signals control expression of the transcription factor Olig3 and the specification of spinal cord neurons. Dev Biol. 2007:303(1):181-90.

8. Le Dreau G, Garcia-Campmany L, Rabadan MA, Ferronha T, Tozer S, Briscoe J, et al. Canonical BMP7 activity is required for the generation of discrete neuronal populations in the dorsal spinal cord. Development. 2012;139:259-68.

9. Burstyn-Cohen T, Kalcheim C. Association between the cell cycle and neural crest delamination through specific regulation of G1/S transition. Dev Cell. 2002;3:383-95.

10. Kahane N, Kalcheim C. Identification of early postmitotic cells in distinct embryonic sites and their possible roles in morphogenesis. Cell Tissue Res. 1998:294:297-307

11. Bronner-Fraser M, Fraser SE. Cell lineage analysis reveals multipotency of some avian neural crest cells. Nature. 1988:335:161-4.

12. Krispin S, Nitzan E, Kassem Y, Kalcheim C. Evidence for a dynamic spatiotemporal fate map and early fate restrictions of premigratory avian neural crest. Development. 2010;137:585-95.

13. Nitzan E, Krispin S, Pfaltzgraff ER, Klar A, Labosky P, Kalcheim C. A dynamic code of dorsal neural tube genes regulates the segregation between neurogenic and melanogenic neural crest cells. Development. 2013;140:2269-79.

14. Liem Jr KF, Tremml G, Jessell TM. A role for the roof plate and its resident TGFb-related proteins in neuronal patterning in the dorsal spinal cord. Cell. 1997;91:127-38.

15. Liem Jr KF, Tremml G, Roelink H, Jessell TM. Dorsal differentiation of neural plate cells induced by BMP-mediated signals from epidermal ectoderm. Cell. 1995;82:969-79.

16. Sela-Donenfeld D, Kalcheim C. Regulation of the onset of neural crest migration by coordinated activity of BMP4 and Noggin in the dorsal neural tube. Development. 1999:126:4749-62.

17. Burstyn-Cohen T, Stanleigh J, Sela-Donenfeld D, Kalcheim C. Canonical Wnt activity regulates trunk neural crest delamination linking BMP/noggin signaling with G1/S transition. Development. 2004;131(21):5327-39.

18. Tozer S, Le Dreau G, Marti E, Briscoe J. Temporal control of BMP signalling determines neuronal subtype identity in the dorsal neural tube. Development. 2013;140(7):1467-74.

19. Le Dreau G, Marti $E$. The multiple activities of BMPs during spinal cord development. Cell Mol Life Sci. 2013;70(22):4293-305.

20. Chizhikov W, Millen KJ. Control of roof plate development and signaling by $\mathrm{Lm \times 1b}$ in the caudal vertebrate CNS. J Neurosci. 2004; 24(25):5694-703.

21. Chizhikov W, Millen KJ. Control of roof plate formation by Lmx1a in the developing spinal cord. Development. 2004;131(11):2693-705.

22. Baek JH, Hatakeyama J, Sakamoto S, Ohtsuka T, Kageyama R. Persistent and high levels of Hes1 expression regulate boundary formation in the developing central nervous system. Development. 2006;133(13):2467-76.

23. Kobayashi T, Kageyama R. Expression dynamics and functions of Hes factors in development and diseases. Curr Top Dev Biol. 2014;110:263-83.

24. Cruz C, Ribes V, Kutejova E, Cayuso J, Lawson V, Norris D, et al. Foxj1 regulates floor plate cilia architecture and modifies the response of cells to sonic hedgehog signalling. Development. 2010;137:4271-82.

25. Shoval I, Ludwig A, Kalcheim C. Antagonistic roles of full-length $\mathrm{N}$-cadherin and its soluble BMP cleavage product in neural crest delamination. Development. 2007;134:491-501

26. Larkins CE, Aviles GD, East MP, Kahn RA, Caspary T. Arl13b regulates ciliogenesis and the dynamic localization of Shh signaling proteins. Mol Biol Cell. 2011:22(23):4694-703.

27. Duband JL, Thiery JP. Distribution of laminin and collagens during avian neural crest development. Development. 1987;101:461-78.

28. Monsonego-Ornan E, Kosonovsky J, Bar A, Roth L, Fraggi-Rankis V, Simsa S, et al. Matrix metalloproteinase $9 /$ gelatinase $B$ is required for neural crest cell migration. Dev Biol. 2012;364(2):162-77.

29. Krishan K, McKinnell I, Patel K, Dhoot GK. Dynamic Id2 expression in the medial and lateral domains of avian dermamyotome. Dev Dyn. 2005;234(2):363-70.

30. Yokota Y, Mori S, Narumi O, Kitajima K. In vivo function of a differentiation inhibitor, Id2. IUBMB Life. 2001:51(4):207-14.

31. Martinsen BJ, Bronner-Fraser M. Neural crest specification regulated by the helix-loop-helix repressor Id2. Science. 1998:281:988-91.

32. Ying QL, Nichols J, Chambers I, Smith A. BMP induction of Id proteins suppresses differentiation and sustains embryonic stem cell self-renewal in collaboration with STAT3. Cell. 2003;115:281-92. 
33. Applebaum M, Ben-Yair R, Kalcheim C. Segregation of striated and smooth muscle lineages by a Notch-dependent regulatory network. BMC Biol. 2014;12:53.

34. Le Dreau G, Saade M, Gutierrez-Vallejo I, Marti E. The strength of SMAD1/5 activity determines the mode of stem cell division in the developing spinal cord. J Cell Biol. 2014;204:591-605.

35. Lopes SS, Yang X, Muller J, Carney TJ, McAdow AR, Rauch GJ, et al. Leukocyte tyrosine kinase functions in pigment cell development. PLoS Genet. 2008;4:e1000026.

36. Simoes-Costa M, Tan-Cabugao J, Antoshechkin I, Sauka-Spengler T, Bronner ME. Transcriptome analysis reveals novel players in the cranial neural crest gene regulatory network. Genome Res. 2014;24:281-90.

37. Panchision D, Pickel J, Studer L, Lee S, Turner P, Hazel T, et al. Sequential actions of BMP receptors control neural precursor cell production and fate. Genes Dev. 2001;15:2094-110.

38. Groysman M, Shoval I, Kalcheim C. A negative modulatory role for Rho and Rho-associated kinase signaling in delamination of neural crest cells. Neural Dev. 2008;3:27.

39. Chizhikov W, Millen KJ. Roof plate-dependent patterning of the vertebrate dorsal central nervous system. Dev Biol. 2005:277(2):287-95.

40. Helms AW, Johnson JE. Specification of dorsal spinal cord interneurons. Curr Opin Neurobiol. 2003;13(1):42-9.

41. von Bubnoff A, Cho KW. Intracellular BMP signaling regulation in vertebrates: pathway or network? Dev Biol. 2001;239(1):1-14.

42. de Pater E, Ciampricotti M, Priller F, Veerkamp J, Strate I, Smith K, et al. Bmp signaling exerts opposite effects on cardiac differentiation. Circ Res. 2012;110(4):578-87.

43. Nitzan E, Pfaltzgraff ER, Labosky PA, Kalcheim C. Neural crest and Schwann cell progenitor-derived melanocytes are two spatially segregated populations similarly regulated by Foxd3. Proc Natl Acad Sci U S A. 2013;110:12709-14.

44. Dessaud E, Ribes V, Balaskas N, Yang LL, Pierani A, Kicheva A, et al. Dynamic assignment and maintenance of positional identity in the ventral neural tube by the morphogen sonic hedgehog. PLoS Biol. 2010;8:e1000382.

45. Sela-Donenfeld D, Kalcheim C. Inhibition of noggin expression in the dorsal neural tube by somitogenesis: a mechanism for coordinating the timing of neural crest emigration. Development. 2000;127:4845-54.

46. Sela-Donenfeld D, Kalcheim C. Localized BMP4-noggin interactions generate the dynamic patterning of noggin expression in somites. Dev Biol. 2002;246:311-28.

47. Hata A, Lagna G, Massague J, Hemmati-Brivanlou A. Smad6 inhibits BMP/ Smad1 signaling by specifically competing with the Smad4 tumor suppressor. Genes Dev. 1998;12:186-97.

48. Linker C, Stern CD. Neural induction requires BMP inhibition only as a late step, and involves signals other than FGF and Wnt antagonists. Development. 2004:131:5671-81.

49. Jouve C, Palmeirim I, Henrique D, Beckers J, Gossler A, Ish-Horowicz D, et al. Notch signalling is required for cyclic expression of the hairy-like gene HES1 in the presomitic mesoderm. Development. 2000;127(7):1421-9.

50. Bai G, Sheng N, Xie Z, Bian W, Yokota Y, Benezra R, et al. Id sustains Hes1 expression to inhibit precocious neurogenesis by releasing negative autoregulation of Hes1. Dev Cell. 2007;13:283-97.

51. Thomas AJ, Erickson CA. FOXD3 regulates the lineage switch between neural crest-derived glial cells and pigment cells by repressing MITF through a non-canonical mechanism. Development. 2009;136:1849-58.

52. Ribes V, Balaskas N, Sasai N, Cruz C, Dessaud E, Cayuso J, et al. Distinct Sonic Hedgehog signaling dynamics specify floor plate and ventral neuronal progenitors in the vertebrate neural tube. Genes Dev. 2010;24:1186-200.

53. Yamauchi K, Phan KD, Butler SJ. BMP type I receptor complexes have distinct activities mediating cell fate and axon guidance decisions. Development. 2008;135(6):1119-28.

54. Shoval I, Kalcheim C. Antagonistic activities of Rho and Rac GTPases underlie the transition from neural crest delamination to migration. Dev Dyn. 2012;241:1155-68.

55. Broom ER, Gilthorpe JD, Butts T, Campo-Paysaa F, Wingate RJ. The roof plate boundary is a bi-directional organiser of dorsal neural tube and choroid plexus development. Development. 2012;139(22):4261-70.

56. Hirata H, Tomita K, Bessho Y, Kageyama R. Hes1 and Hes3 regulate maintenance of the isthmic organizer and development of the mid/ hindbrain. EMBO J. 2001;20(16):4454-66.

57. Shimojo H, Ohtsuka T, Kageyama R. Oscillations in notch signaling regulate maintenance of neural progenitors. Neuron. 2008;58(1):52-64.
58. Kageyama R, Ohtsuka T, Kobayashi T. The Hes gene family: repressors and oscillators that orchestrate embryogenesis. Development. 2007:134(7):1243-51.

59. Murata K, Hattori M, Hirai N, Shinozuka Y, Hirata H, Kageyama R, et al. Hes1 directly controls cell proliferation through the transcriptional repression of p27Kip1. Mol Cell Biol. 2005;25(10):4262-71.

60. Castella P, Sawai S, Nakao K, Wagner JA, Caudy M. HES-1 repression of differentiation and proliferation in PC12 cells: role for the helix 3-helix 4 domain in transcription repression. Mol Cell Biol. 2000;20(16):6170-83.

61. Dessaud E, Yang LL, Hill K, Cox B, Ulloa F, Ribeiro A, et al. Interpretation of the sonic hedgehog morphogen gradient by a temporal adaptation mechanism. Nature. 2007:450:717-20.

62. Dottori M, Gross MK, Labosky P, Goulding M. The winged-helix transcription factor Foxd3 suppresses interneuron differentiation and promotes neural crest cell fate. Development. 2001;128:4127-38.

63. Zou H, Choe KM, Lu Y, Massagué J, Niswander L. BMP signaling and vertebrate limb development. Cold Spring Harbor Symp Quant Biol. 1997;62:269-72.

64. Stephen LA, Davis GM, McTeir KE, James J, McTeir L, Kierans M, et al. Failure of centrosome migration causes a loss of motile cilia in talpid(3) mutants. Dev Dyn. 2013;242(8):923-31.

65. Lecoin L, Sii-Felice K, Pouponnot C, Eychene A, Felder-Schmittbuhl MP. Comparison of maf gene expression patterns during chick embryo development. Gene Expr Patterns. 2004;4(1):35-46.

\section{Submit your next manuscript to BioMed Central and we will help you at every step:}

- We accept pre-submission inquiries

- Our selector tool helps you to find the most relevant journal

- We provide round the clock customer support

- Convenient online submission

- Thorough peer review

- Inclusion in PubMed and all major indexing services

- Maximum visibility for your research

Submit your manuscript at www.biomedcentral.com/submit
) Biomed Central 